\title{
Ernährung und Rheuma
}

Patienten mit rheumatischen Erkrankungen setzen grosse Hoffnungen auf eine Linderung ihrer Beschwerden durch eine spezielle Ernährung. Wissenschaftliche Belege, dass dies teilweise von Erfolg gekrönt ist, sind in letzter Zeit veröffentlicht worden.

Viele Patienten, die an Rheuma leiden, versuchen mit einer Umstellung der Ernährung den Krankheitsverlauf zu beeinflussen. Vor allem rheumatische Erkrankungen, die eine Infektion als Ursache haben, können teilweise durch die Nahrung beeinflusst werden. $\mathrm{Zu}$ vermeiden sind Stoffe, die Entzündungen fördern. Patienten sollen davon profitieren, die Zufuhr an Arachidonsäure zu senken, aus der im weiteren Stoffwechsel Prostaglandine gebildet werden, die wiederum entzündungsfördernd wirken. Der menschliche Körper bildet zwar Arachidonsäure zum grössten Teil selbst, jedoch ist sie in Fleisch und Eiern in grösserer Konzentration vorhanden, sodass die Rheumaliga den Patienten empfiehlt, nur 1-3 Eier pro Woche $\mathrm{zu}$ essen. Andere Nahrungsmittel sollen in den Entzündungsstoffwechsel hemmend eingreifen: ungesättigte Omega-3-Fettsäuren, Antioxidantien, die Vitamine E und C, Beta-Carotinoide oder Zink und Selen. Die Omega3-Fettsäuren greifen z.B. in den Stoffwechsel der Prostaglandine ein und scheinen auch noch über andere Mechanismen entzündungshemmend zu wirken.

Die wissenschaftliche Evidenz für den Einfluss der Ernährung auf den Verlauf von rheumatischer Arthritis ist erst in den letzten Jahren erarbeitet worden. In einer Literaturübersicht versuchten Stamp et al. [1] den Forschungsstand zusammenzufassen. In den von ihnen analysierten Studien zeigte sich, dass sich bei Patienten, die mehr n-3-Fettsäure zu sich nahmen, eine leichte Verbesserung ihrer Symptome zeigte. Bei einer kleinen An- zahl von Patienten führte auch Fasten, ein Umstellen auf eine vegane Ernährung und der Verzicht auf bestimmte Lebensmittel zu einer Verbesserung. $\mathrm{Ob}$ sich diese Methoden jedoch im Allgemeinen eignen, Patienten mit rheumatischer Arthritis zu behandeln, bezweifeln die Autoren. Sie seien zu aufwendig oder zu schwierig, um sie über einen längeren Zeitraum beizubehalten.

Untersuchungen haben sich mit der Frage beschäftigt, ob das Risiko, an rheumatischer Arthritis zu erkranken, auch von der Ernährung abhängt. Ein sehr grosser Einfluss ist nicht zu erwarten, da eine grosse genetische Komponente als Risikofaktor erkannt wurde: Das HLA-DRB1x0404-Allel und das Tyrosin-Phosphatase-Gen PTPN 22 sind die wichtigsten genetischen Faktoren. Daneben spielen eine Reihe von MHC-Genen und jene für den Tumor-Nekrose-Faktor eine Rolle. Weibliche Geschlechtshormone haben eine schützende Wirkung, während Infektionen etwa mit dem Eppstein-Barr-Virus, dem Parvovirus oder einigen Bakterien wie Proteus oder Mycoplasma die rheumatische Arthritis begünstigen.

In einer kleinen Literaturstudie mit 14 Arbeiten haben Pattison et al. [2] eine Schutzwirkung einiger Substanzen gefunden: Wer viel Olivenöl sowie Fisch mit hohem Ölgehalt und Gemüse und Nahrung, die reich an BetaCryptoxanthin ist, zu sich nimmt, ist besser gegen rheumatische Arthritis geschützt. In 3 Studien liess sich ein Zusammenhang mit einem niedrigen Serumspiegel an Antioxidantien mit einem erhöhten Risiko für die Erkran- kung finden. Die Mittelmeerdiät soll ebenfalls eine schützende Wirkung haben [3].

Fischöl scheint nach den Ergebnissen einer epidemiologischen Studie eine leichte Schutzwirkung zu haben. Rosell et al. [4] konnten ein verringertes Risiko in Abhängigkeit von Fischöl in der Nahrung finden.

In einer grossen randomisierten Doppelblindstudie, an der sich mehr als 40000 Frauen beteiligten, untersuchten Karlson et al. [5] die Möglichkeit, mit Vitamin E das Risiko zu senken. Sie konnten allerdings keine signifikante Reduktion in der Gruppe der Teilnehmerinnen finden, die mehr Vitamin E zu sich nahm.

Wichtige Risikofaktoren, die man durch eine Umstellung der Ernährung und eine gesündere Lebensführung ausschalten kann, sind ein hoher Kaffeekonsum und das Rauchen [6]. (rfi)

\section{Literatur}

Stamp LK, Michael JJ, Cleveland LG: Diet and rheumatoid arthritis: a review of the literature. Semin Arthritis Rheum 2005;35:77-94.

2 Pattison DJ, Harrison RA, Symmons DPM: The role of diet in susceptibility to rheumatoid arthritis: a systematic review. J Rheumatol 2004; 31:1310-1319.

3 Sales, C, Oliviero F, Spinella P: The mediterranean diet model in inflammatory rheumatic diseases (article in Italian). Reumatismo 2009; 61:10-14.

4 Rosell M, Wesley AM, Rydin K, Klareskog L, Alfredsson L; EIRA study group: Dietary fish and fish oil and the risk of rheumatoid arthritis. Epidemiology 2009;20:896-901.

5 Karlson EW, Shadick NA, Cook NR, Buring JE, Lee IM: Vitamin $\mathrm{E}$ in the primary prevention of rheumatoid arthritis: the Women's Health Study. Arthritis Rheum 2008;259:1589-1595.

6 Oliver JE, AJ Silman: Risk factors for the development for rheumatoid arthritis. Scand J Rheumatol 2006;35:169-174.

\section{KARGER}

